\title{
GNSS Network RTK Regional Ionospheric Modelling Studies And Performance Analysis
}

\author{
Weiming Tang, Lei Jin, Jianhui Cui, Chuang Shi and Yongfeng Zhang \\ (GNSS Research Center, Wuhan University) \\ (Positioning Attitude Navigation Direction and Application, PANDA) \\ (E-mail: shi@whu.edu.cn)
}

\begin{abstract}
In this paper the Grid correction method (GRID) was proposed for the first time as an interpolation method of the double differenced ionospheric delay for GNSS Network RTK. The Distance Interpolation Method (DIM), Linear Interpolation Method (LIM), the Kriging Interpolation Method (KRG) and the GRID are described and compared in the aspect of their interpolation performance. It was shown in the tests that the GRID interpolation method performed better than the other three. For mid-latitude stations, its interpolation accuracy is better than $1 \mathrm{~cm}$, and the extrapolation accuracy is better than $2 \mathrm{~cm}$. Furthermore, the GRID method can achieve a good performance even at low satellite elevation, and also its interpolation accuracy can be better than $2 \mathrm{~cm}$ at low latitudes.
\end{abstract}

\section{KEYWORDS}

1. Network RTK. 2. Double differenced ionospheric delay. 3. Grid correction method.

Submitted: 25 June 2014. Accepted: 29 July 2015. First published online: 7 September 2015.

1. INTRODUCTION. Considering the distance-dependent system error in a certain area that has a strong spatial correlation, the main purpose of Network Real Time Kinematic (NRTK) is to apply a certain interpolation algorithm to interpolate or extrapolate the system error at the rover station, so as to achieve a precise positioning. Therefore it is of great importance to estimate the system error precisely at the rover station, because this not only affects the reliability and success rate of the ambiguity solving, but also affects the positioning accuracy (Ge et al., 2010; Zou, 2013). Among different kinds of errors influencing RTK positioning, ionospheric error plays an important role in the positioning accuracy and reliability (Tang et al., 2007; Cao et al., 2013). A good interpolation method could estimate the system error correction accurately for the rover, thereby reducing the ambiguity resolution time. Scholars have carried out a wide range of studies on this subject and proposed a variety of interpolation algorithms (Zhang, 2010), such as the Linear Interpolation Model (LIM) (Wanninger, 1995), the Low order Surface fitting Model (LSM) (Dai et al., 2001) and Distance Interpolation Method (DIM) (Gao et al., 
1997). This paper will introduce a new interpolation algorithm, the Grid correction method (GRID). The introduction is followed by the comparison with the DIM, the LIM and the KRG, in the aspect of the interpolation performance. Analysis and discussion on their performance are then given.

\section{DOUBLE DIFFERENCED IONOSPHERIC DELAY BETWEEN REFERENCE}

STATIONS. The double differenced observation equations can be written as:

$$
\begin{aligned}
& \lambda_{1} \Delta \nabla \varphi_{1}=\Delta \nabla \rho-\lambda_{1} \Delta \nabla N_{1}-k_{2} \Delta \nabla I+\Delta \nabla \zeta_{\text {trop }} \\
& \lambda_{2} \Delta \nabla \varphi_{2}=\Delta \nabla \rho-\lambda_{2} \Delta \nabla N_{2}-k_{1} \Delta \nabla I+\Delta \nabla \zeta_{\text {trop }}
\end{aligned}
$$

where $k_{i}=f_{i}^{2} /\left(f_{1}^{2}-f_{2}^{2}\right) ; \Delta \nabla \varphi_{\mathrm{i}}$ is the measured double differenced carrier phase; $\Delta \nabla \rho$ is the true double differenced geometric range; $\Delta \nabla N_{\mathrm{i}}$ is the double differenced carrier phase ambiguity; $\Delta \nabla \zeta_{\text {trop }}$ is the double differenced troposphere delay; $\Delta \nabla I$ is the double differenced ionosphere delay. After the integer ambiguities between reference stations are fixed, the noise of double differenced carrier phase measurement is so small that it can be ignored. Once the ambiguities are correctly resolved, the centimetre-level accuracy of the double differenced ionospheric measurement can be derived through a combination of the dual-frequency carrier phase measurements as follows,

$$
\Delta \nabla I=\left(\lambda_{1} \Delta \nabla \varphi_{1}-\lambda_{2} \Delta \nabla \varphi_{2}\right)+\left(\lambda_{1} \Delta \nabla N_{1}-\lambda_{2} \Delta \nabla N_{2}\right)
$$

3. NETWORK RTK INTERPOLATION ALGORITHM. Since the correlation of the atmospheric errors gets weaker as the baseline distance increases, these errors cannot be eliminated cleanly in the double differencing, so long-range NRTK positioning requires the support of the atmospheric correction, where the correction for the ionospheric error is of the most important influence. This work conducted an analysis on the commonly used DIM, LIM and KRG methods. Then the method introduced by Liao (2000), which uses the double differenced observations to establish a regional level ionospheric grid model, was applied to the network RTK ionospheric interpolation, and named as the Grid correction method (GRID).

3.1. Distance Interpolation Method (DIM). This method uses the information generated by the reference stations and computes a set of interpolation coefficients to obtain the double differenced delay at the rover station (Gao et al., 1997). The interpolation coefficient is a function of the distances between the reference stations and the rover station. The double differenced ionospheric delay at the rover station can be obtained as,

$$
\Delta \nabla I=\sum_{i=1}^{n-1} \frac{c_{i}}{c} \Delta \nabla I_{i}
$$

where $n-1$ is the total number of auxiliary reference stations; $c_{i}$ is the distance between the auxiliary reference station $i$ and the rover station and $\mathrm{c}=\sum_{i=1}^{n-1} c_{i} ; \Delta \nabla I_{i}$ is the double differenced ionospheric delay between the auxiliary reference station $i$ and master reference station. 
3.2. Linear Interpolation Model (LIM). This method was first proposed by Wanninger (1995). Since the double differenced ionospheric delay $\Delta \nabla I$ is a linear function of the plane coordinate differences, it can be expressed as follows,

$$
\begin{aligned}
\Delta \nabla I & =\frac{\partial \Delta \nabla I\left(X_{K}-X_{M}\right)}{\partial X}+\frac{\partial \Delta \nabla I\left(Y_{K}-Y_{M}\right)}{\partial Y} \\
& =a\left(X_{K}-X_{M}\right)+b\left(Y_{K}-Y_{M}\right)
\end{aligned}
$$

The coefficients $a$ and $b$ in Equation (4) can be obtained by the least squares method when there are three reference stations in a certain area. With the known coefficients $a$ and $b$, the double differenced ionospheric delay at the rover can be computed.

3.3. Kriging Interpolation Method (KRG). A method which fully takes the spatial correlation between given points into account to predict the value for a point in between is the Kriging Interpolation Method. According to this method, the interpolated value at the rover can mathematically be given as (Odijk, 2000; Zhang, 2010):

$$
\Delta \nabla I_{u}=\left(C_{i u}^{T} \cdot C_{i}^{-1}-\lambda e_{n}^{T} C_{i}^{-1}\right) \Delta \nabla I_{i}, \text { where } \lambda=\left(e_{n}^{T} C_{i}^{-1} e_{n}\right)^{-1}\left(C_{i u}^{T} C_{i}^{-1} e_{n}-1\right)
$$

In Equation (5) $u$ represents the rover station, $i$ is the base station and $n$ is the total number of reference stations; $C_{i u}=\left[\begin{array}{c}c_{u 1} \\ c_{u 2} \\ \vdots \\ c_{u n}\end{array}\right], C_{i}=\left[\begin{array}{cccc}c_{11} & c_{12} & \cdots & c_{1 n} \\ c_{21} & c_{22} & \cdots & c_{2 n} \\ \vdots & \vdots & \ddots & \vdots \\ c_{n 1} & c_{n 2} & \cdots & c_{n n}\end{array}\right], \Delta \nabla I_{i}=\left[\begin{array}{c}\Delta \nabla I_{1 n} \\ \Delta \nabla I_{2 n} \\ \vdots \\ \Delta \nabla I_{n n}\end{array}\right], \Delta \nabla I_{i}$ is the double differenced ionospheric delay between the base station $i$ and master reference station and $\Delta \nabla I_{n n}=0$. The scalar $c_{r 1 r 2}$ denotes the covariance function between locations $\mathrm{r} 1$ and $\mathrm{r} 2$, and needs to be specified to perform the interpolation. In this article, the covariance function is chosen as a simple linear function of the distance:

$$
c_{r 1 r 2}=l_{\max }-l_{r 1 r 2}
$$

where $l_{r 1 r 2}[\mathrm{~km}]$ denotes the distance between locations $\mathrm{r} 1$ and $\mathrm{r} 2 ; l_{\max }$ is a certain maximum distance $[\mathrm{km}]$. With respect to the choice of $l_{\max }$, in order to guarantee that the matrix $c_{i}$ is positive-definite, it should be larger than the longest distance between the base stations.

3.4. Grid correction method (GRID). This interpolation algorithm follows the method proposed by Liao (2000), which uses the double differenced ionospheric delays of the reference stations to establish a regional level ionospheric grid model. This method was only previously used for the recovery of the total electron content. In this paper, the GRID method was proposed for the first time as an interpolation method of the double differenced ionospheric delay for GNSS Network RTK. The double differenced ionospheric delay for the rover can be interpolated from this grid model. Each double differenced ionospheric delay obtained by Equation (2) can be converted to the Slant Total Electron Content (STEC) at four Ionosphere Piercing Points (IPP). In order to recover the vertical TEC at the grid points, the mapped vertical TEC measurement at each IPP needs to be linked with the vertical TEC at the surrounding four grid points, as shown in Figure 1.

In this work, the following model has been adopted to establish the functional relationship between the double differenced ionospheric measurements and the vertical 


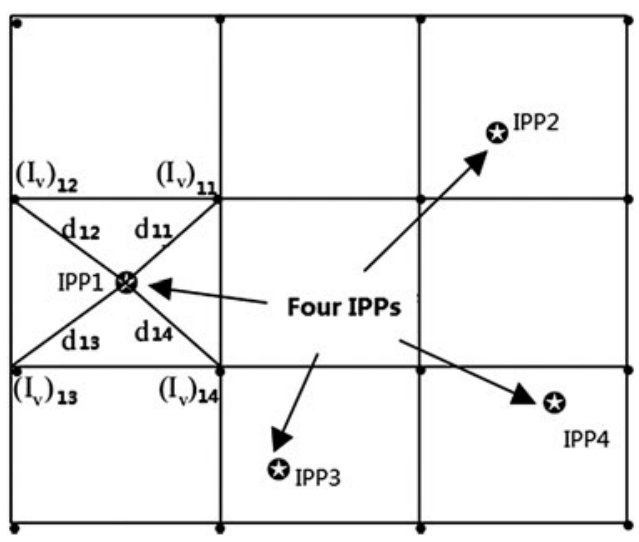

Figure 1. Diagram of the grid model.

TEC over the surrounding grid points (Liao, 2000; Zhao, 2003):

$$
\begin{aligned}
\Delta \nabla I & =\frac{40.28\left(f_{1}^{2}-f_{2}^{2}\right)}{f_{1}^{2} f_{2}^{2}} S T E C_{i j}^{p q}=I_{j}^{q}-I_{i}^{q}-I_{j}^{p}+I_{i}^{p} \\
& =f\left(e_{1}\right) \sum_{n=1}^{4}\left(I_{v}\right)_{1 n} \cdot w_{1 n}-f\left(e_{2}\right) \sum_{n=1}^{4}\left(I_{v}\right)_{2 n} \cdot w_{2 n} \\
& -f\left(e_{3}\right) \sum_{n=1}^{4}\left(I_{v}\right)_{3 n} \cdot w_{3 n}+f\left(e_{4}\right) \sum_{n=1}^{4}\left(I_{v}\right)_{4 n} \cdot w_{4 n}
\end{aligned}
$$

where $i$ stands for the master reference station; $j$ stands for another auxiliary reference station; $p$ is the reference satellite, $q$ is the observed satellite; $f(e)$ is the slant factor which maps the STEC to the Vertical Total Electron Content (VTEC); $\left(I_{v}\right)_{i j}$ is the VTEC at the associate grid points; $w_{m n}$ is the weighting function. There are several weighting functions available, but the analysis from El-Arini et al. (1994) indicated that there is little difference in accuracy between these weighting functions. The inverse distance weighting function was chosen here and it is expressed as Equation (8), where $m$ represents the corresponding IPP, $n$ for the corresponding grid point:

$$
w_{m n}=\frac{1}{d_{m n}} / \sum_{k=1}^{4} \frac{1}{d_{m k}}
$$

Using Equations (7) and (8) and the least squares adjustment, the VTEC value at each grid point can be obtained, which could lead to the establishment of the regional ionospheric grid model. Also in accordance with Equation (7), the double differenced ionospheric delay for the rover can be calculated using this GRID model. This interpolation method is invariant to the choice of the master reference station because the parameters in this method are the absolute instead of the relative value of total electron content.

This method fully uses the information of each IPP. When IPPs of different satellites fall in the same grid, they could complement each other, rather than simply 


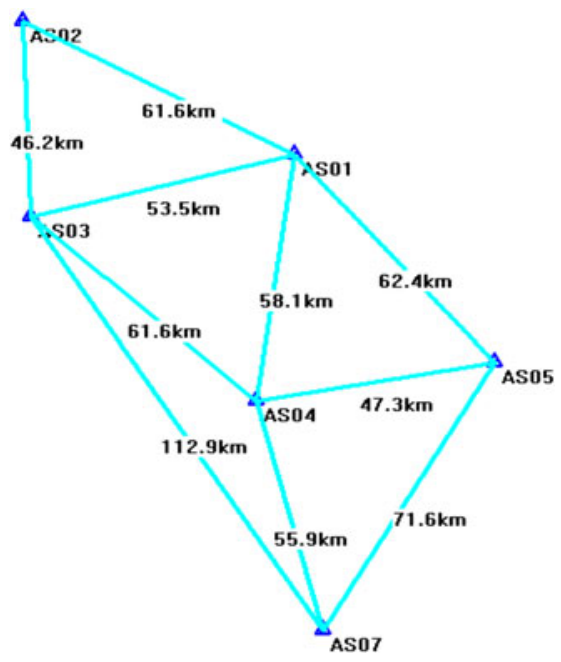

Figure 2. Distribution of stations in Anshan, China.

interpolating between the same satellites. Meanwhile, the tracking of the observable satellites could assist in computing the double differenced ionospheric delay of the newly rising satellites. In addition, when there are enough reference stations, a high spatial resolution for a regional network could follow the rapid ionosphere variation over the area. This could guarantee the high accuracy of the double differenced ionospheric delay correction.

4. TEST VALIDATION AND ANALYSIS. To analyse the performance of the above four methods, tests were conducted using Global Positioning System (GPS) datasets in three networks in China. After the integer ambiguities between reference stations are fixed, the centimetre-level accuracy of the double differenced ionospheric delays between the auxiliary reference stations station and the master reference station can be obtained, which were treated as real measurements. The Root Mean Square (RMS) is calculated by omitting one reference station from the network and comparing the interpolation results for all satellites at that station with the real measurements at each epoch.

4.1. Case Study 1. A case study was carried out with the stations illustrated in Figure 2. These stations are located in Anshan, China and their mean latitude is $40 \cdot 80^{\circ}$. The data was collected on the Day of Year (DoY) 8/2013. A satellite elevation cut-off angle of $10^{\circ}$ was used for analyses and the observation interval is one second. AS01 is the master reference station. The double differenced ionospheric delays (from the master reference station to the auxiliary station) for AS02, AS04 and AS07 are presented in Figure 3, the horizontal axis is the GPS time and the vertical axis is the double differenced ionospheric delay, in units of metres. Each colour denotes a specific satellite and the same colour in different plots denotes the same Satellite Vehicle (SV). It can be seen from Figure 3 that the values of the double differenced ionospheric delay are in the range of $-0 \cdot 4 \sim 0 \cdot 4$ metres. The longer the baseline length, the greater the absolute value of delay is. This is because with the baseline increasing, the spatial 

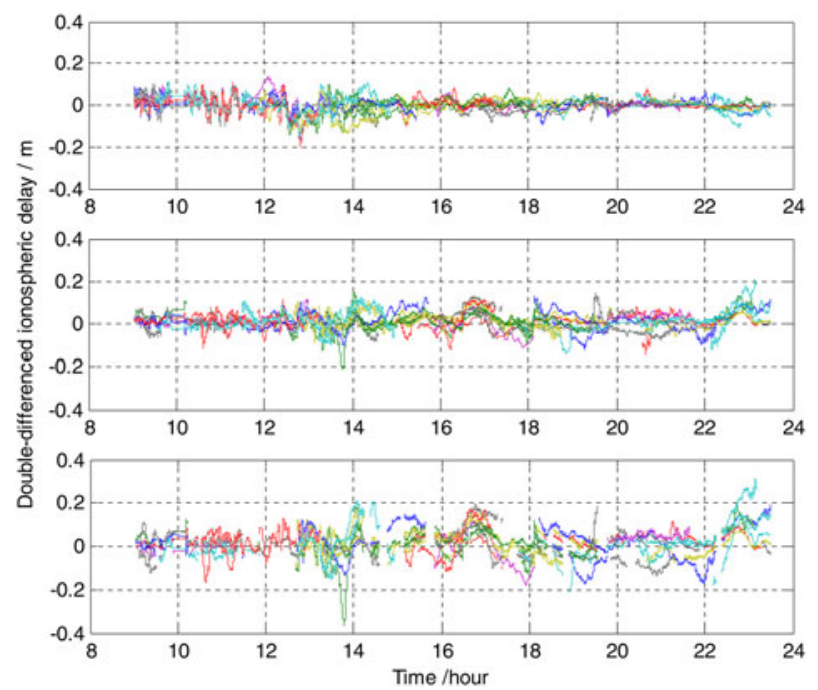

Figure 3. Double differenced ionospheric delay.

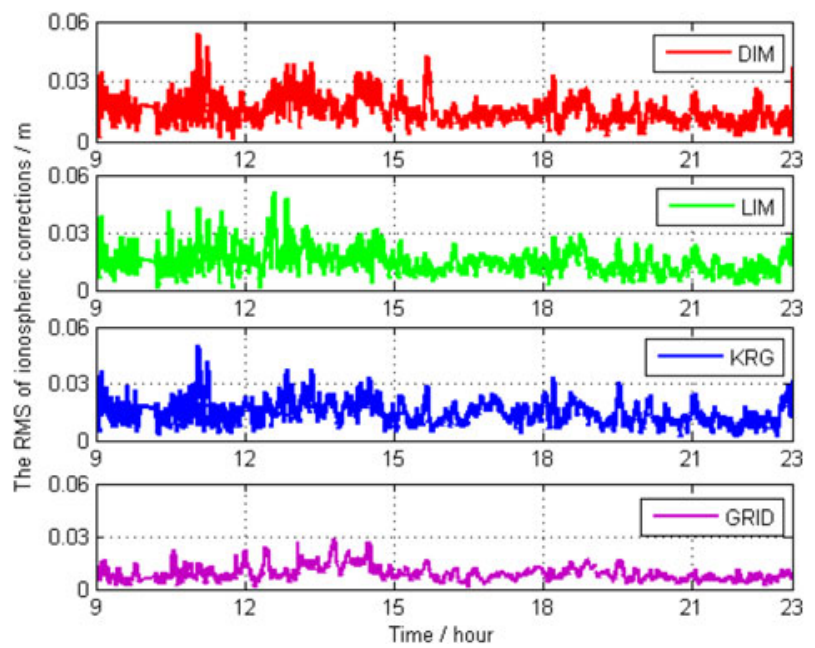

Figure 4. Comparison among four algorithms for ionospheric corrections within the network.

correlation will be getting weaker. This case study compared the above-mentioned four methods in two cases; the rover station is located inside and outside the network separately. The height of the single-layer ionosphere is set to be $450 \mathrm{~km}$. According to the distribution of IPPs in this case, the grid size is configured as $2^{\circ} \times 4^{\circ}$ latitude by longitude.

4.1.1. The rover station is inside the network. AS04 is selected as the rover in this case. The interpolation results were evaluated with the truth values, and the RMS of each epoch was used to illustrate the accuracy of each method, as shown in Figure 4. The RMSs of each epoch were averaged and listed in Table 1. It can be 
Table 1. The mean RMS of three algorithms (unit: $\mathrm{m}$ ).

\begin{tabular}{lllll}
\hline Location of rover station & DIM & LIM & KRG & GRID \\
\hline AS04(inside) & $0 \cdot 0151$ & $0 \cdot 0146$ & 0.0144 & $0 \cdot 0089$ \\
AS02(outside) & 0.0332 & 0.0201 & 0.0201 & 0.0111 \\
\hline
\end{tabular}

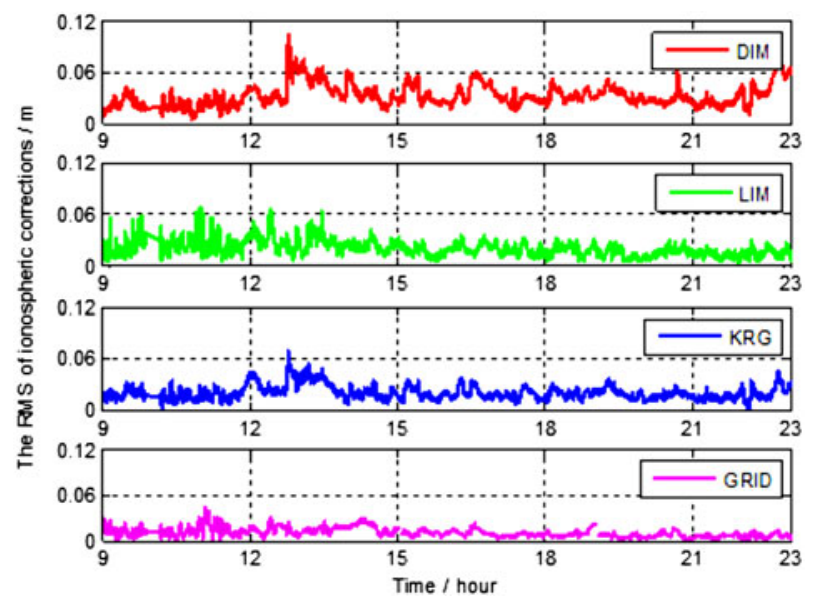

Figure 5. Comparison among four algorithms for ionospheric corrections beyond the network.

seen that when the rover station is inside the network, the accuracy of the GRID method is superior to the other three methods; the accuracies of LIM and KRG are close to each other while the performance of DIM is the worst.

4.1.2. The rover station is outside the network. $\mathrm{AS} 02$ is selected as the rover station, which is located outside the network. A similar statistical analysis was carried out for AS02 as well, as shown in Figure 5. The mean RMS value was listed in Table 1. It can be seen that when the rover station is outside the network, the accuracy of the GRID method is better than the other three methods.

4.1.3. Analysis. In the above test results, the GRID algorithm performs best. This method can achieve good results no matter whether the rover is inside the network or not. The GRID algorithm is less affected by the position of the rover station because it makes full use of the information of each IPP. It is also reflected in Figures 4 and 5 that KRG and LIM outperform DIM because these two methods take the directionality of the double differenced ionospheric delay into consideration. To further explain the difference in performances, the relationship between the interpolation results and elevation angles is also shown in the following. PRN 11 and PRN 16 observed by AS02 are selected for illustration. Considering that the DIM showed the poorest interpolation results and the accuracies of LIM and KRG are close to each other, LIM and GRID are chosen as examples. The results presented in Figures 6 and 7 (left: GRID; right: LIM). In the comparison for PRN16, it could be found that at around 19:00 the LIM method cannot estimate the double differenced ionospheric delay because the surrounding reference stations failed to observe this satellite. However the GRID method is still able to estimate 

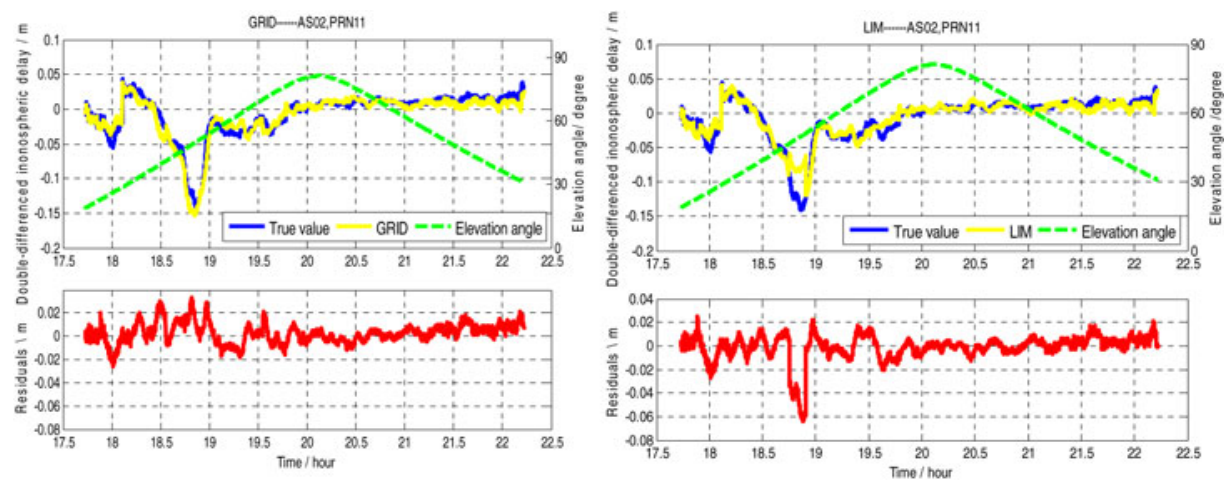

Figure 6. Comparison of a single satellite interpolation results (PRN11).
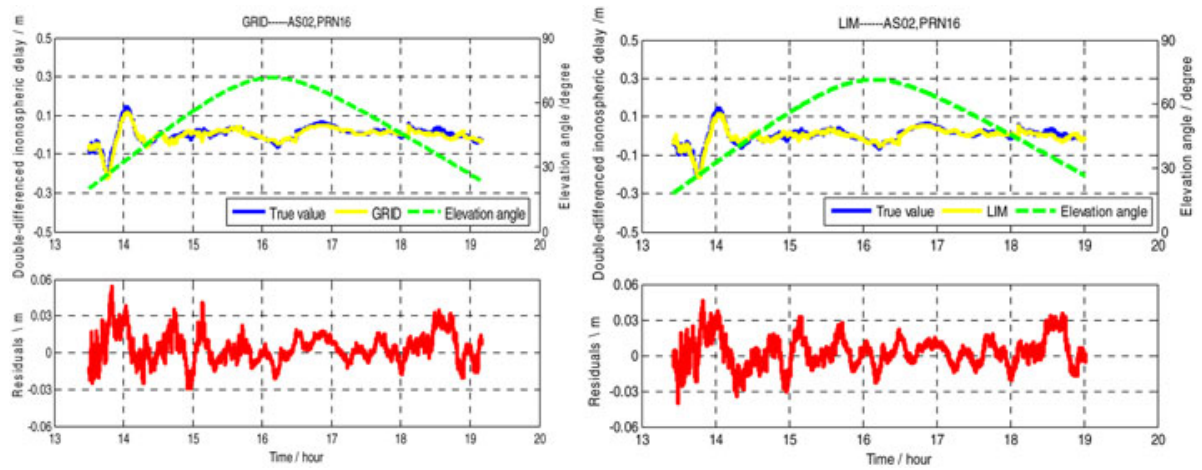

Figure 7. Comparison of a single satellite interpolation results (PRN16).

the double differenced ionospheric delay by using the IPP information from other satellites. It also can be seen from both figures that the GRID interpolation can maintain good performance even when the satellite elevation angle is low. This further illustrates that the GRID interpolation method is more robust and it can provide a higher interpolation precision.

4.2. Case Study 2. The activity level of free electrons in the ionosphere is closely related to the latitude. With the latitude decreasing, the ionosphere becomes more active. In order to analyse the performance of the four methods in low latitudes, this paper chose the data collected from a Continuously Operating Reference Station (CORS) network in Guangdong (southern China), as the second example. The mean latitude of the stations is $22.98^{\circ}$. Distribution of these stations with baseline length is shown in Figure 8. GPS data of Day of Year (DoY) 146/2014 for a 24 hour span was processed by the methods described above. The height of the singlelayer ionosphere is set to be $450 \mathrm{~km}$. A satellite elevation cut-off angle of $10^{\circ}$ was used for analyses and the observation interval is one second.

This example chose station ZQGT as the master reference station. Due to the dramatic effect of the free electrons, the values of the double differenced ionospheric delay 


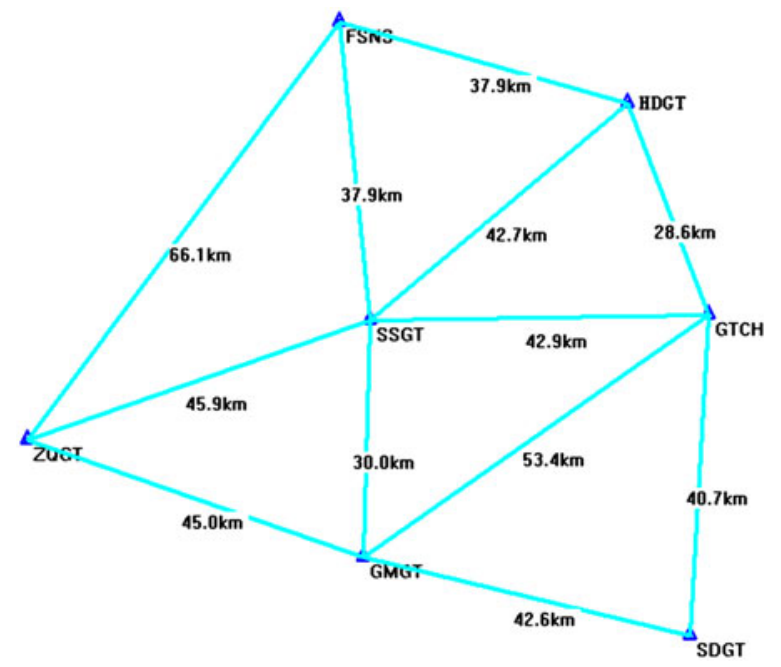

Figure 8. Distribution of the stations.

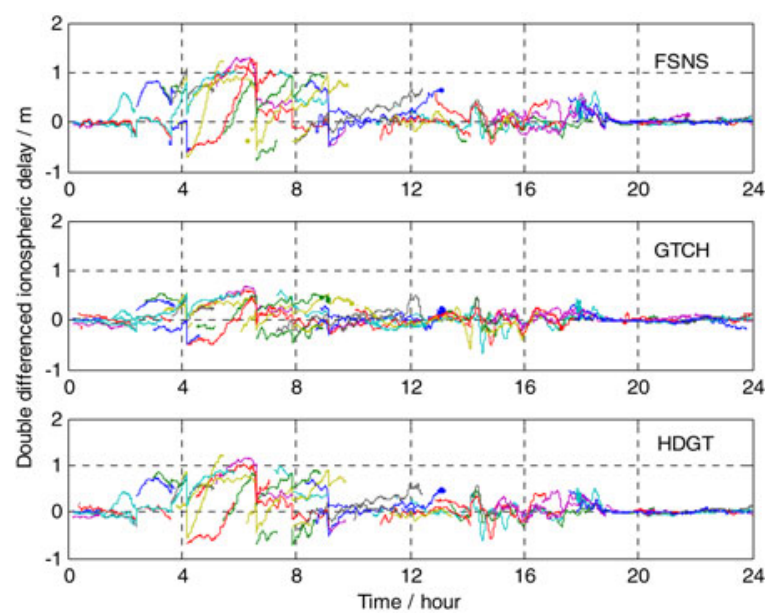

Figure 9. Double differenced ionospheric delay.

are in the range of $-1 \cdot 0 \sim 2.0$ metres while the baseline length is about $40 \mathrm{~km}$. The double differenced ionospheric delay of three stations is shown in Figure 9. The horizontal axis denotes the GPS time and the vertical axis denotes the double-differenced ionospheric delay in the unit of metres. Each colour denotes a specific satellite and the same colour in different plots denotes the same SV.

4.2.1. The rover station is inside the network. In this case, the SSGT station was selected as the rover station and the interpolation results from each method were compared with the truth values. The RMSs at each epoch were used to illustrate the interpolation accuracy of each method. Regarding the distribution of the IPPs, the latitude 
Table 2. The mean RMS of three algorithms (unit: $\mathrm{m}$ ).

\begin{tabular}{lllll}
\hline Location of rover station & DIM & LIM & KRG & GRID \\
\hline SSGT (inside) & 0.0520 & $0 \cdot 0194$ & 0.0227 & $0 \cdot 0161$ \\
SDGT (outside) & 0.0498 & 0.0258 & 0.0280 & 0.0197 \\
\hline
\end{tabular}

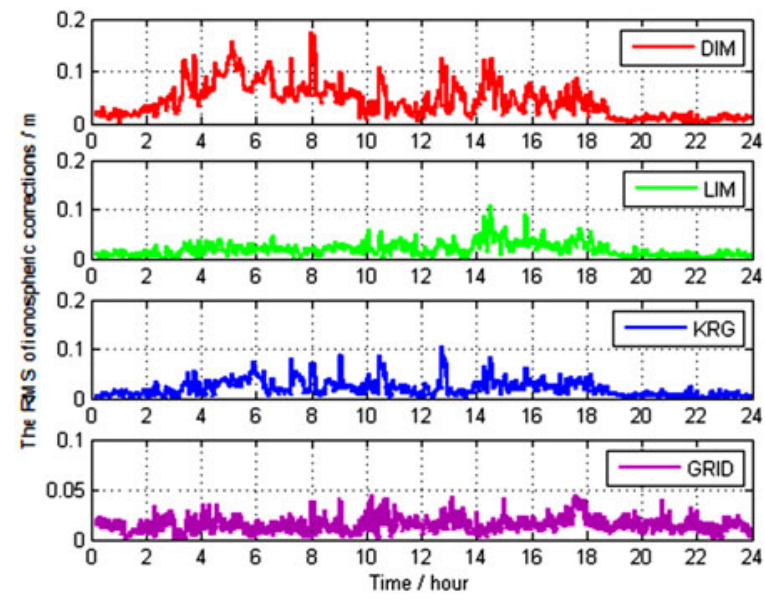

Figure 10. Comparison among four algorithms for ionospheric corrections within the network.
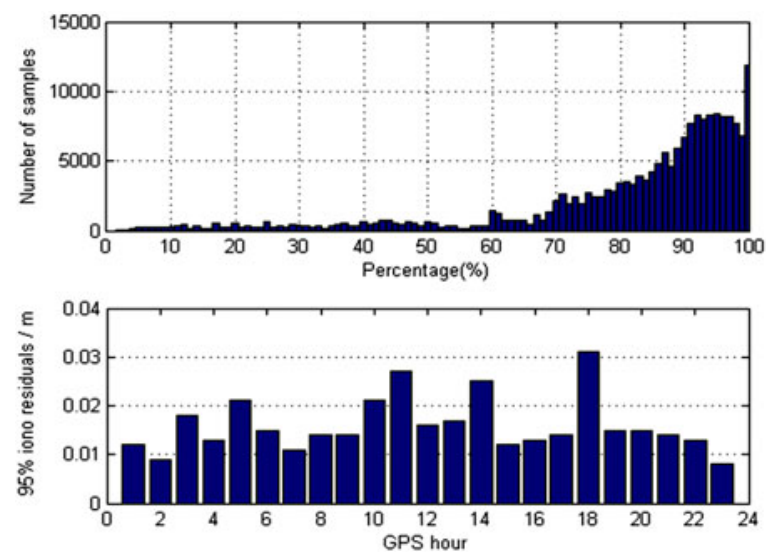

Figure 11. Correction percentage of GRID method (top) and IRIM for ZQGT—SSGT (below).

is in the range of 9 to $33^{\circ}$ and the longitude is in the range of 100 to $130^{\circ}$ while the grid resolution is $1^{\circ} \times 2^{\circ}$ latitude by longitude in the GRID algorithm. The RMSs of each epoch were averaged, as shown in Table 2. Figure 10 shows the RMS against time. It can be seen that the maximum RMS of GRID is less than $0.05 \mathrm{~m}$, and this is superior to the other three methods. Figure 11 showed the percentage of correction using GRID method and also reflected that the GRID could achieve more than $70 \%$ accuracy 


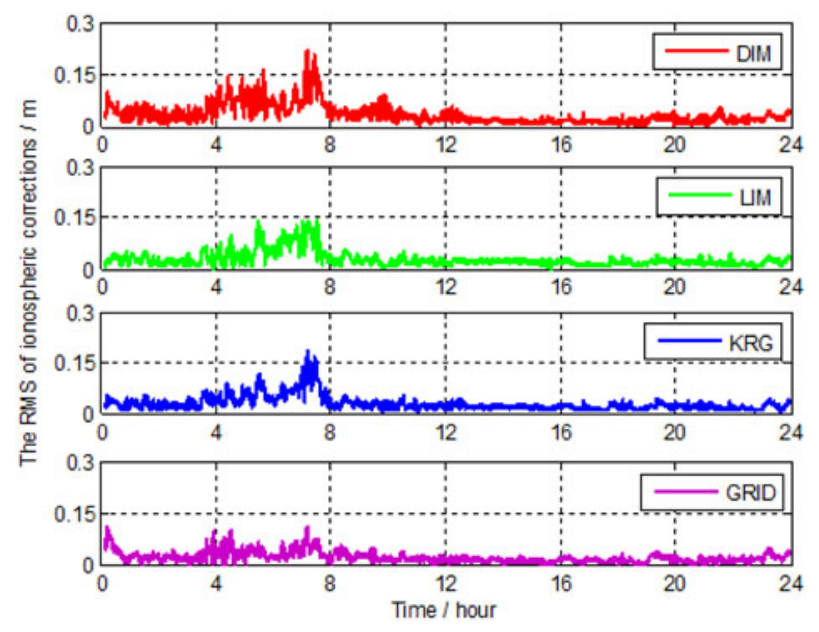

Figure 12. Comparison among four algorithms for ionospheric corrections beyond the network.

interpolation. The IRIM (Ionospheric Residual Integrity Monitoring, IRIM) indicator (Chen et al., 2003) using GRID was also shown in Figure 11 and this indicator described the residual remaining in the baseline from ZQGT to SSGT of whole day.

4.2.2. The rover station is outside the network. SDGT is selected as the rover station located outside the network. A similar statistical analysis was carried out, as shown in Figure 12. The mean RMS value is listed in Table 2. It can be observed that when the rover station is outside the network, the accuracy of the GRID method is still superior to that of the other three methods.

4.3. Case Study 3. In this case, all the five stations, which are part of Zhongshan CORS, are located in the south of China and the mean latitude is $22 \cdot 50^{\circ}$. The distribution of these stations is shown in Figure 13. The distances between the reference stations are also shown in this figure. DACH was selected as the master reference station and SHIJ as the rover. GPS data of Day of Year (DoY) 158/2014 for a 24 hours span was processed. The height of the single-layer ionosphere is set to be $450 \mathrm{~km}$. A satellite elevation cut-off angle of $10^{\circ}$ was used for analyses and the observation interval is one second.

The double differenced ionospheric delays of GPS for each station are shown in Figure 14, with the horizontal axis is the GPS time and the vertical axis is in the units of metres. Each colour denotes a specific satellite and the same colour in different plots denotes the same SV. The values of the double differenced ionospheric delay can be observed in the range of $-0.8 \sim 0.8$ metres. Figure 15 shows the RMS against time. The mean RMS value is $0.0443 \mathrm{~m}$ for DIM, $0.0213 \mathrm{~m}$ for LIM, $0.0200 \mathrm{~m}$ for KRG and $0.0186 \mathrm{~m}$ for GRID. The PRN5 observed by the rover (SHIJ) was selected for illustrated the relationship between the interpolation results and elevation angles. Considering that the DIM showed poorest in interpolation results and the accuracies of LIM and KRG are close to each other, so LIM and GRID are chosen as examples. The results are presented in Figure 16 (left: GRID; right: LIM). 


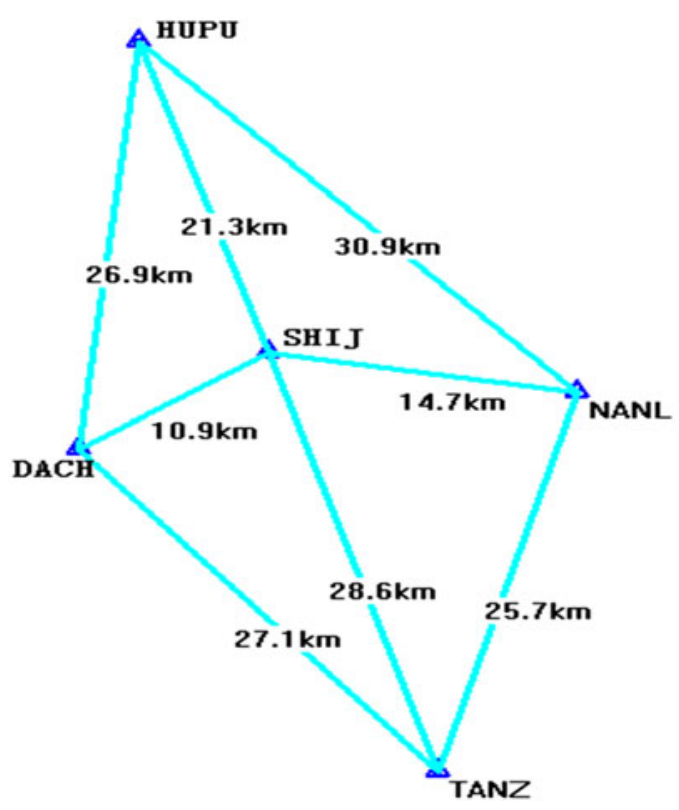

Figure 13. Distribution of stations in Zhongshan, China

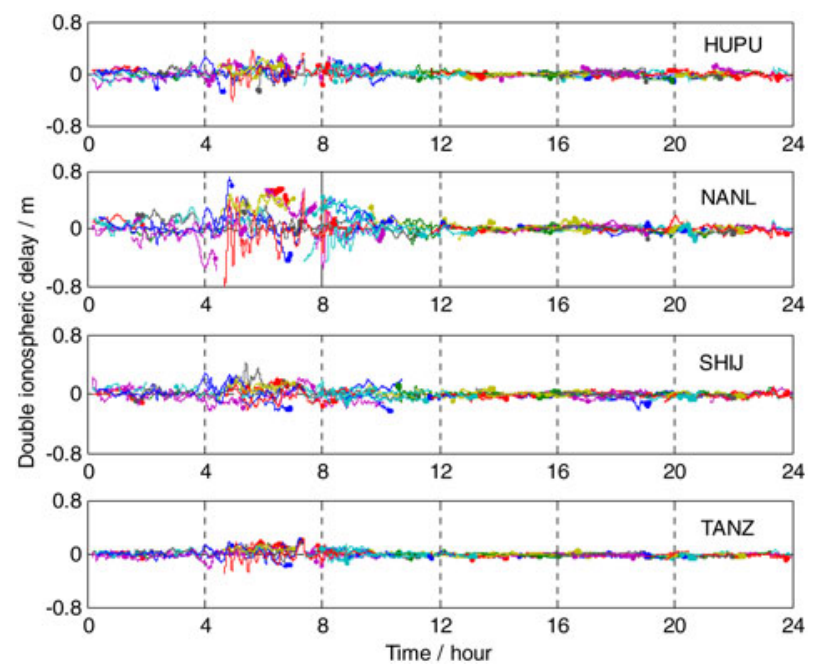

Figure 14. Double differenced ionospheric delay.

5. CONCLUSIONS. In this paper, the Distance Interpolation Method (DIM), linear interpolation (LIM), the Kriging interpolation method (KRG) and the GRID correction method (GRID) were described and compared with each other on their interpolation performance. The results showed that the GRID algorithm performs better 


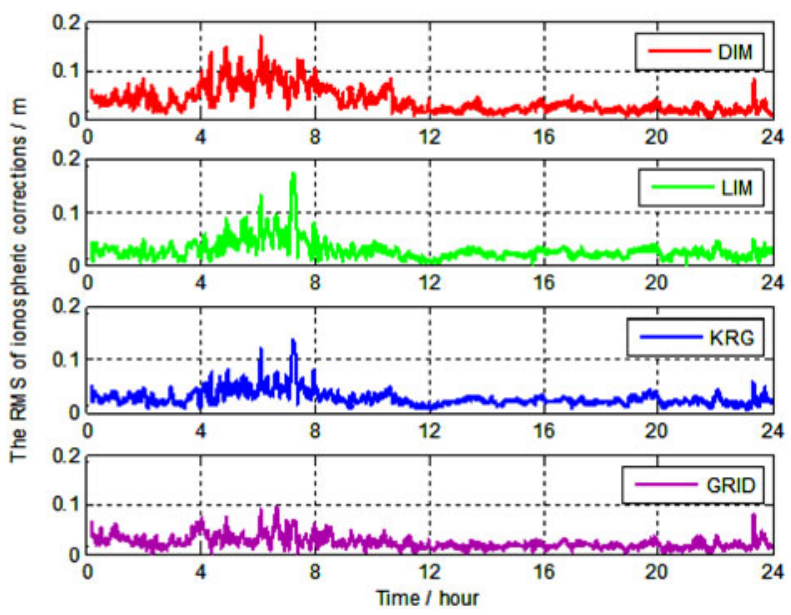

Figure 15. Comparison among four algorithms for ionospheric corrections.
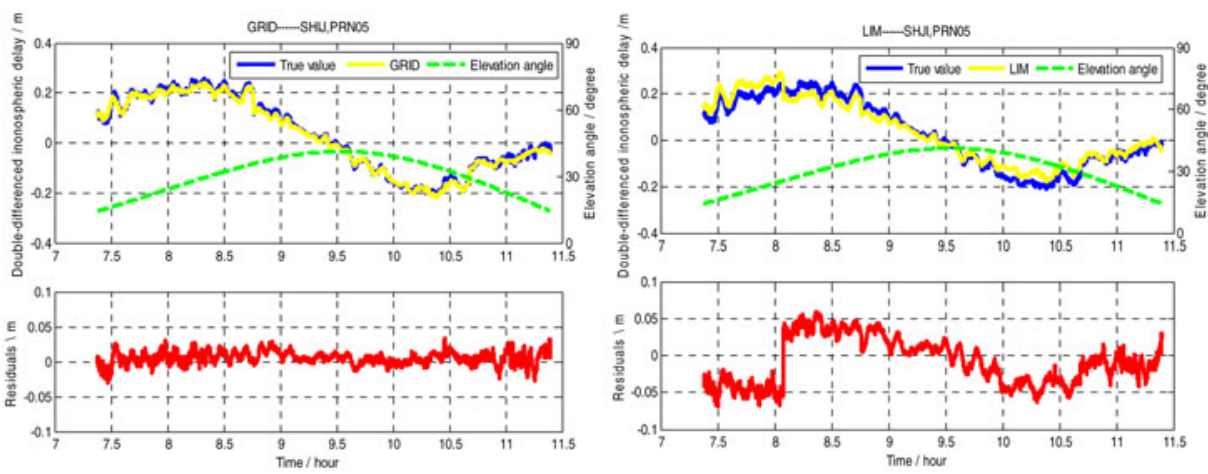

Figure 16. Comparison of a single satellite interpolation results (PRN05).

than the other three, for the reason that it fully uses the information of each IPP. When IPPs of different satellites fall in the same grid, they can complement each other, rather than being interpolated separately. In addition, when the satellite elevation angle is low, the results of GRID could still achieve a high accuracy. LIM and KRG outperform DIM because these two methods take the directionality of the double differenced ionospheric delay into consideration. DIM interpolation can obtain good results when the rover is located inside the network, but its accuracy becomes poorer when the rover station is outside the network.

\section{ACKNOWLEDGEMENTS}

This work is partially sponsored by China 863 program (Grant No. 2014AA123101/01 and 2012AA12A202), the Natural Science Foundation of China (Grant No. 41004014) and partially supported by the National Science Fund for Distinguished Young Scholars (Grant No. 41325015) as well as the Fundamental Research Funds for the Central Universities (No. 2012214020208). 


\section{REFERENCES}

Cao, W., Guo, J., Xie, X. and Zhou, M. (2013). Modeling Method of Ionospheric Delay Based on CORS. Journal of Geomatics, 38(3), 12-16 (in Chinese).

Chen, X., Landau, H. and Vollath, U. (2003). New tools for Network RTK integrity monitoring. Proceedings of IONGPS/GNSS 2003, Portland, Oregon, Sept. 9-12, 1355-1361.

Dai, L., Han, S., Wang, J. and Rizos, C. (2001). A study on GPS/GLONASS multiple reference station techniques for precise real-time carrier phase-based positioning. ION GPS2001. Salt Lake City: Palm Springs.

El-Arini, M.B., Hegarty, C.J., Fernow, J.P. and Klobuchar, J.A. (1994). Development of an Error Budget for a GPS Wide-Area Augmentation System (WAAS). Proceedings of the 1994 National Technical Meeting of The Institute of Navigation, San Diego, CA, January, 927-936.

Gao, Y., Li, Z. and McLellan, J.F. (1997). Carrier phase based regional area differential GPS for decimeterlevel positioning and navigation. 10th International Technical Meeting of the Satellite Division of the Institute of Navigation. Kansas City, Missouri, 16-19 September, 1305-1313.

Ge, M., Zou, X. and Dick, G. (2010). An alternative Network RTK approach based on undifferenced Observation corrections. ION GNSS, USA.

Liao, X. (2000). Carrier Phase Based Ionosphere Recovery over a Regional Area GPS Network. Dissertation. The University Of Calgary.

Odijk, D. (2000). Fast precise GPS positioning in the presence of ionospheric delays. Dissertation. Delft University of Technology.

Tang, W., Liu, J. and Liu, H. (2007). A Modified Combined Bias Interpolation Method for GNSS Network RTK. Geomatics and Information Science of Wuhan University, 12, 1156-1159 (in Chinese).

Wanninger, L. (1995). Improved ambiguity resolution by regional differential modelling of the ionosphere. 8th International Technical Meeting of the Satellite Division of the Institute of Navigation, Palm Springs, California, 12-15 September, 55-62.

Zhang, S. (2010). The GPS/GLONASS Integrated CORS Network Atmosphere Modeling and RTK Algorithm Implementation. Dissertation, School of Geodesy and Geomatics, Wuhan University (in Chinese).

Zhao, X. (2003). Research On the Methods of Establishing Grid Ionosphere Model Over A Regional Area GPS Network. Dissertation. School of Geodesy and Geomatics, Wuhan University (in Chinese).

Zou, X., Feng, Y., Tang, W., and Liu, J. (2013). Equivalence of undifferenced HiRIM method and the existing double-differenced network RTK methods. Geomatics and Information Science of Wuhan University, 38, 1276-1280 (in Chinese). 